\title{
251. Some Existence Theorems in Cluster Set Theory
}

\author{
By Shinji Yamashita \\ Mathematical Institute, Tôhoku University \\ (Comm. by Kinjirô Kunugr, M. J. A., Dec. 12, 1970)
}

1. Let $C$ be the unit circle and $D$ be the open unit disk in the complex plane.

Theorem 1. There exists a holomorphic function $f$ in $D$ for which the set $I(f)$ of Plessner points [3, p. 147] is residual [3, p. 75] on $C$ and of logarithmic measure [7, p. 64] zero.

Theorem 2. There exists a bounded univalent holomorphic function $f$ in $D$ for which the set $M(f)$ of Meier points [3, p. 153] is of logarithmic measure zero.

Furthermore, we obtain some improvements of Bagemihl-Seidel's results [2, p. 191, Corollaries $3 \sim 5]$, one of which may be stated as

Theorem 3. There exist a holomorphic function $f$ in $D$ and $a$ subset $S$ of $C$, being of logarithmic measure zero, such that the radial cluster set [3, p. 72] of $f$ at any point of $C-S$ coincides with the unit circle.

Remark 1. A bounded set of logarithmic measure zero is known to be of logarithmic capacity zero. In Remark 3 of the next section we ascertain this for our special example $S$.

I wish to express my warmest thanks to Prof. K. Hatano for valuable conversations.

2. We construct a subset $S$ of $C$ satisfying the following three conditions :

(i ) $C-S$ is of first Baire category on $C$.

(ii) $S$ is a $G_{\delta}$ subset of $C$.

(iii) The logarithmic measure of $S$ is zero.

Let $K=\left\{z_{1}, \cdots, z_{n}, \cdots\right\}$ be a countable dense subset of $C$ and let $\varepsilon_{1}, \cdots, \varepsilon_{k}, \cdots$ be a sequence of positive numbers such that $\varepsilon_{k} \rightarrow 0$ as $k \rightarrow \infty$. Let $\delta_{k n}$ be an open disk containing $z_{n}$ whose radius is $r_{k n}=\exp \left(-2^{n} / \varepsilon_{k}\right)(k, n=1,2, \cdots)$. Let $\delta_{k}=\bigcup_{n=1}^{\infty} \delta_{k n}$ and let $\delta=\bigcap_{k=1}^{\infty} \delta_{k}$. Then $S=\delta \cap C$ is the desired one. Indeed, for any $k$, the closed set $C-\delta_{k}$ is nowhere dense on $C$ since $\delta_{k} \cap C \supset K$ is open and dense on $C$. Therefore the set

$$
\text { (*) } \quad C-S=\bigcup_{k=1}^{\infty}\left(C-\delta_{k}\right)
$$

is of first category on $C$. To prove (iii) we use the same notation as in [7, p. $63 \mathrm{ff}$.$] with h(t)=\{\log (1 / t)\}^{-1}$. We use "disks" instead of 
"squares" in p. 63 of [7]; this is not an essential change. Then, since $\delta_{k n}(n=1,2, \cdots)$ cover $S$ and $r_{k n}<\exp \left(-1 / \varepsilon_{k}\right) \equiv \rho_{k}$, we have

$$
H\left(\rho_{k}\right)(\text { for } S) \leqq \sum_{n=1}^{\infty} h\left(r_{k n}\right)=\sum_{n=1}^{\infty}\left(\varepsilon_{k} / 2^{n}\right)=\varepsilon_{k} .
$$

This means that $H\left(\rho_{k}\right) \rightarrow 0$ as $k \rightarrow \infty$, which, combined with $\rho_{k} \rightarrow 0$ as $k \rightarrow \infty$, proves that the logarithmic measure of $S$ is zero.

Remark 2. Generalizations to higher dimensional spaces or to metric spaces under the condition that $C$ is a perfect set in the considered spaces are fairly easy. Prof. Hatano communicated orally that the condition (iii) may well be generalized to "Hausdorff measure".

Remark 3. By Erdös-Gillis's theorem [7, p. 66] combined with (iii) the inner logarithmic capacity and hence the logarithmic capacity of $S$ is zero since $S$ is capacitable by (ii). Consequently our example is simpler and more general than Kishi-Nakai's [4].

3. By Lappan's theorem [5, Theorem 2] there exists a holomorphic function $f$ in $D$ for which $I(f)=S$ since $S$ is a $G_{\delta}$ subset of $C$. This proves Theorem 1 .

We apply Bagemihl's theorem [1] to the set $C-S$. Then there exists a bounded univalent holomorphic function $f$ in $D$ such that $M(f) \cap(C-S)=\emptyset$ (empty). Theorem 2 follows from $M(f) \subset S$ and (iii).

Since $C-S$ is $F_{\sigma}$ of first category and of the form of (*) on $C$ we have the improvements of Corollaries $3 \sim 5$ in [2, p. 191] in the sense that the subsets of $C$ of exceptional character are of logarithmic measure zero.

Remark 4. Bagemihl's proof of his theorem in [1] depends in part on a theorem of Lohwater and Piranian [6, p. 7, Theorem 1']. We remark that some theorems in [6] may well be applied to our $F_{\sigma}$ set $C-S$. For example, we have:

There exists a bounded univalent holomorphic function $f$ in $D$ such that $f$ has a radial limit $f(\zeta)$ at every point $\zeta \in C$ and that the function $f(\zeta)$ on $C$ is discontinuous at every $\zeta \in C-S$ and is continuous at every $\zeta \in S$.

\section{References}

[1] F. Bagemihl: On the sharpness of Meier's analogue of Fatou's theorem. Israel J. Math., 4, 230-232 (1966).

[2] F. Bagemihl and W. Seidel: Some boundary properties of analytic functions. Math. Z., 61, 186-199 (1954).

[3] E. F. Collingwood and A. J. Lohwater: The Theory of Cluster Sets. Cambridge (1966).

[4] M. Kishi and M. Nakai: Linear set of the second category with zero capacity. Proc. Japan Acad., 44, 633-635 (1968). 
[5] P. Lappan: A characterization of Plessner points. Bull. London Math. Soc., 2, 60-62 (1970).

[6] A. J. Lohwater and G. Piranian: The boundary behavior of functions analytic in a disk. Ann. Acad. Sci. Fenn. Ser. A I Math., 239, 1-17 (1957).

[7] M. Tsuji: Potential Theory in Modern Function Theory. Tokyo (1959). 